\title{
Liquid crystal dynamic flow control by bidirectional alignment surface
}

\author{
Y. W. Li, C. Y. Lee, and H. S. Kwok ${ }^{\text {a) }}$ \\ Center for Display Research, Hong Kong University of Science and Technology, \\ Clear Water Bay, Hong Kong
}

(Received 30 September 2008; accepted 20 January 2009; published online 10 February 2009)

\begin{abstract}
We investigate the behavior of liquid crystal dynamic flow in a cell with a bidirectional alignment (BDA) surface. Numerical simulations show that with a BDA surface having a pitch comparable to the cell gap $d$, the liquid crystal dynamic flow direction can be controlled by the driving voltage. Such an effect can be applied to bistable twisted nematic displays without the need for anchoring breaking. (C) 2009 American Institute of Physics. [DOI: 10.1063/1.3080198]
\end{abstract}

There are numerous studies of liquid crystal (LC) dynamic flow on homogenous alignment surfaces. Van Doorn et al. ${ }^{1}$ stated that dynamic flow imparts a backward torque on the LC molecules near the center of the device. Berreman et $\mathrm{al}^{2}{ }^{2}$ observed this phenomenon on twisted nematic devices. Bos and Koehler ${ }^{3}$ reported that faster LC display response can be achieved by removing this backflow effect. They suggested that for the bend deformation, the backward torque can be eliminated. For the case of bistable twisted nematic (BTN) devices, dynamic backflow is essential to the switching mechanism. Dozov et ll $^{4}$ proposed a switching mechanism based on asymmetric anchoring alignment (strong polar anchoring on the master plate and weak polar anchoring on the slave plate). When the electric field is turned off rapidly, the master plate director rotates and creates a dynamic flow on the slave plate. Therefore, the cell relaxes rapidly to the transient bend state and then to the $\pi$-twisted state. If the applied electric field decreases slowly, the backflow effect is weak and the device will relax adiabatically to the homogenous state.

It is obvious that the dynamic flow of the LC is very important to determine the dynamic performance of the LC device. In this paper, we propose a new method to control LC flow dynamics using a bidirectional alignment (BDA) layer. In the following, the mechanism of such idea will first be explained. Then, we demonstrate the idea by applying this technology to the BTN device. A new BTN display, called multidomain BTN (MD-BTN), which switches between the no-twist and $\pi$-twisted states, has successfully been fabricated.

The BDA surface is a heterogeneous alignment surface with a periodic pattern. Figure 1 shows the detailed structure of the BDA. It has a periodicity of $L$ and is composed of two types of domains $D_{1}$ and $D_{2} . D_{1}$ and $D_{2}$ have different pretilt angles $\theta_{1}$ and $\theta_{2}$, respectively, on the same plane. The LC molecules are tilted in opposite directions so that $\theta_{1}+\theta_{2}$ is always larger than $90^{\circ}$. It is intuitively obvious that the LC molecules are either tilted at $\theta_{1}$ or $\theta_{2}$, respectively, on the BDA surface, and then relax to a uniform tilt angle in a distance $L_{H}$, which is determined by the pitch $L$. The LC deformation inside the bulk system can be calculated by minimizing the total energy consisting of the bulk elastic energy and surface anchoring energy ${ }^{5}$

${ }^{a)}$ Electronic mail: eekwok@ust.hk.

$$
F_{\mathrm{tot}}(\mathbf{n})=\int_{V} F(\mathbf{n}) d v+\frac{1}{2} \sum_{i} W_{i} \sin ^{2}\left(\theta-\theta_{i}\right),
$$

where $\mathbf{n}$ denotes the LC director, $W_{i}$ is the finite polar surface anchoring energy of the domains, and $F(\mathbf{n})$ is the FrankOseen elastic energy given by

$$
\begin{aligned}
F(\mathbf{n})= & \frac{1}{2}\left[K_{11}(\nabla \cdot \mathbf{n})^{2}+K_{22}\left(\mathbf{n} \cdot \nabla \times \mathbf{n}-q_{o}\right)^{2}+K_{33} \| \mathbf{n} \times \nabla\right. \\
& \left.\times \mathbf{n} \|^{2}\right],
\end{aligned}
$$

where $K_{11}, K_{22}$, and $K_{33}$ are the splay, twist, and bend elastic constants, respectively, and $q_{o}$ is the chiral pitch of the cell. Figure 1 shows the calculated director field for the case of infinitely strong surface anchoring energy, $\theta_{1}=\pi-\theta_{2}$ and $D_{1}=D_{2}=0.5 \mathrm{~L}$. All the material constants used in the numerical calculations refer to MBBA. ${ }^{1}$ Evidently the director near $z=0$ undertakes an abrupt change from $\theta$ to $\pi-\theta$ near the domain boundary and relaxes to $0^{\circ}$ tilt along $+z$. It is also obvious that a uniform deformation can be achieved at some critical position $L_{H}<0.5 L$.

Now suppose the LC cell has a top substrate with a conventional homogeneous alignment and a bottom substrate with a BDA. Let the cell gap $d$ be the same as the pitch $L$. There will be alternating "antiparallel" and "parallel" LC alignments in the LC cell. Since the uniform deformation layer $L_{H}$ is about $d / 2$, there is strong interaction between the two domains. It should be noted that $L_{H}$ is usually much larger than the extrapolation length of $K_{22} / W \approx 10 \mathrm{~nm}$. When an electrical pulse is applied, the induced dynamic flow at the two domains will interact with each other in an interesting manner. To describe the dynamic response of the system, we need two equations. One is the balance of forces acting on the fluid, and the other is the balance of torques

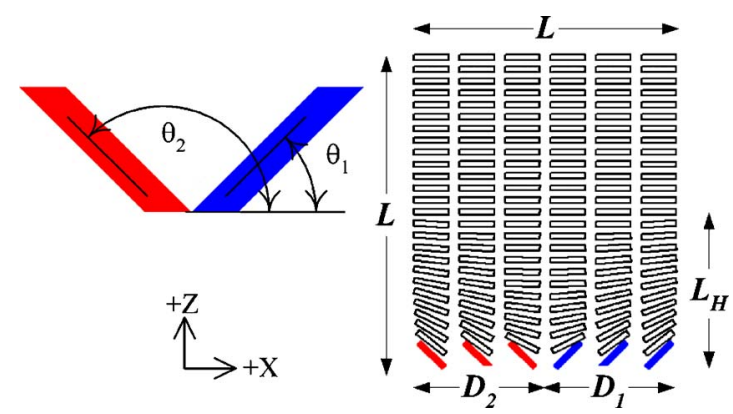

FIG. 1. (Color online) (Left) Pretilt angles and (right) director distribution induced by a BDA surface. 
acting on the director. Fluid inertia can be ignored, provided that the time constant in which acceleration takes place is much larger than the time constant in which the elastic energy relaxes to equilibrium. ${ }^{1}$ We describe the director in the Cartesian coordinate system $\mathbf{n}=\left(\begin{array}{lll}n_{x} & n_{y} & n_{z}\end{array}\right)$ and $|\mathbf{n}|=1$. The Ericksen and Leslie ${ }^{6,7}$ equations of motion for nematic LCs can be simplified to be

$$
\begin{aligned}
& c_{i}=\sigma_{j i}, \\
& 0=G_{i}+g_{i}+\pi_{j i, j},
\end{aligned}
$$

where $i$ and $j$ denote $x, y$, or $z$ components, $\sigma_{j i}$ is the $i$ th component of the surface force acting at the $x_{j}$ plane, and $G_{i}$ is the external body torque. Since there is no transverse electric field, $G_{x}=G_{y}=0, g_{i}$ is the internal body torque, $\pi_{j i}$ is the $i$ th component of the surface torque across the $x_{j}$ plane, and $c_{i}$ is a constant that is obtained by assumption that the velocities of $\mathbf{n}$ at the boundary is zero. It is equivalent to assuming that the anchoring energy is infinitely strong. The terms in Eq. (3) have not been written out in detail but they can be obtained in a straightforward manner. ${ }^{1,2,6-8}$ In the calculations, the optical anisotropy and $d / P$ ratio are $\Delta n$ $=0.1478$ and 0.25 , respectively.

Figure 2 illustrates the results of this simulation to examine the relaxation dynamics of the LC cell when an electrical pulse is applied and turns off sharply. Figure 2(a) shows the molecular alignment for the two cases of strong $(0-100 \mathrm{~ms})$ and weak electrical pulses (100-200 ms). Figures 2(b) and 2(c) show the flow velocity of the LC molecules in the $x$-direction for these two cases. In both cases, there are two dynamic flows coexisting in the system at the moment of turning off the applied electrical pulse. They are the splay flow $(S)$ for the parallel alignment region and homogenous flow $(H)$ for the antiparallel alignment region. It can be seen that the dynamic flows near the upper part $>d / 2$ overlap. At the bottom part of the LC cell, the two flows compete with each other. In Fig. 2(b), for the case of strong electrical pulse, The $H$-flow is totally overtaken by strong $S$-flow at $8 \mathrm{~ms}$. The $S$-flow induces opposite directions of the molecular rotations near the top and bottom alignment boundaries at comparable velocity. The resulting $180^{\circ}$ bend texture relaxes in few milliseconds to the $180^{\circ}$ twist texture $T$.

In Fig. 2(c), for the case of weak electrical pulse, a weak $S$-flow cannot fully overtake the $H$-flow at antiparallel alignment region, as seen at $112 \mathrm{~ms}$. The flow coupling effect on BDA makes the flow velocity of the molecules near the BDA boundary become much slower than those near the top boundary. Because of the elastic coupling, ${ }^{4}$ the molecules on the top boundary and in the middle of the cell rotate in the same direction. The $180^{\circ}$ bend texture, which is induced by the $S$-flow, is then limited at $<d / 2$ region. Recalling that $d / P$ ratio of the LC bulk is 0.25 , the relaxation from $180^{\circ}$ bend to $T$ texture at $<d / 2$ region becomes unfavorable due to insufficient effective $d / P$ ratio. It finally decays to the more stable $U$ texture along $H$-flow direction. Figure 2(d) shows the effect of the tilt angles on the flow control. As the tilt angle increases, the curvature elastic coupling effect becomes stronger while the hydrodynamic coupling ${ }^{4}$ effect is depressed. Therefore, if the tilt angle is too low, the $S$-flow will always dominate the relaxation. For the high tilt angle case, $S$-flow will become too weak to overtake the $H$-flow.
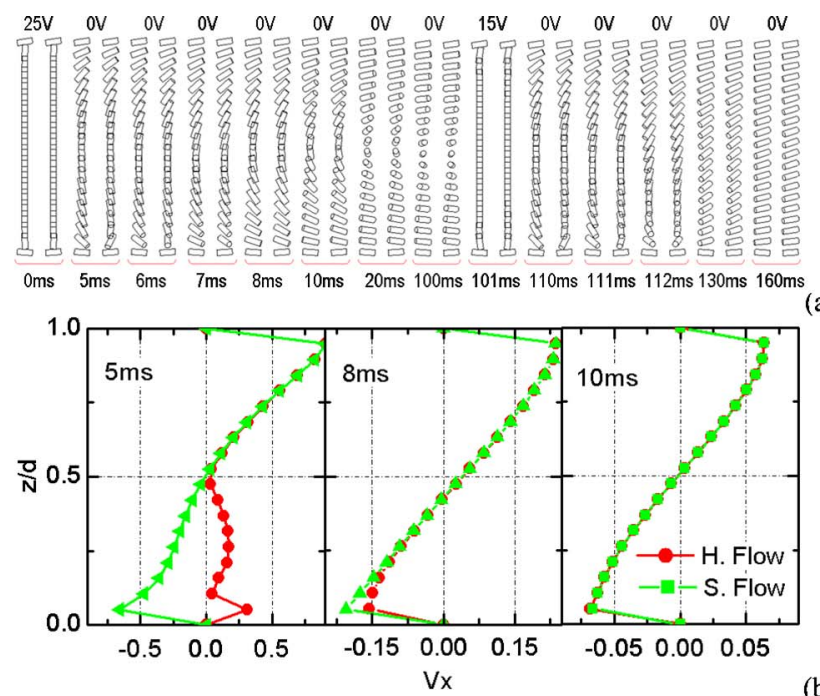

(a)

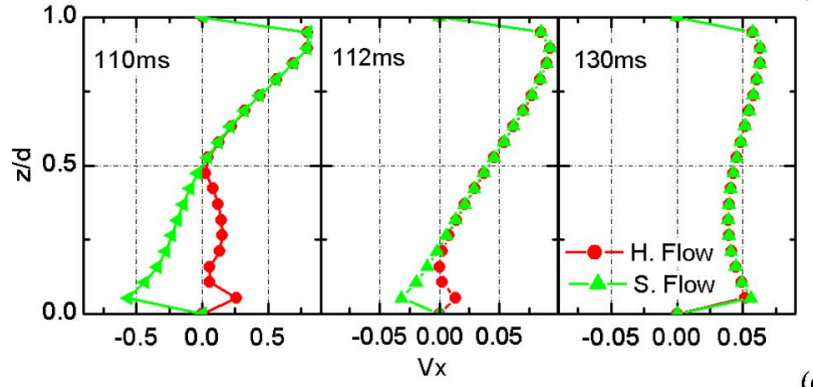

(c)
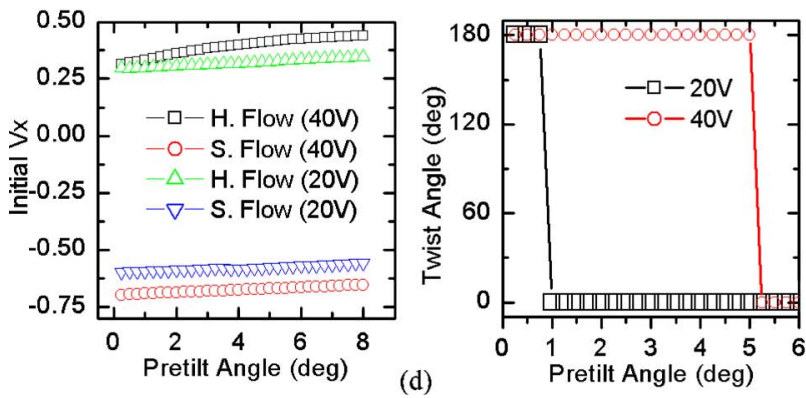

(e)

FIG. 2. (Color online) (a) Simulated LC molecule distribution as a function of time for high voltage (0-100 ms) and low voltage driving (100-200 ms). (b) Flow velocity distribution in $x$ direction for high voltage case. Left: at $5 \mathrm{~ms}$. Middle: at $8 \mathrm{~ms}$. Right: at $10 \mathrm{~ms}$. (c) Flow velocity distribution in $x$ direction for low voltage case. Left: at 110 ms. Middle: at 112 ms. Right: at $130 \mathrm{~ms}$. (d) Dependence of initial flow velocity $V_{x}$ on BDA pretilt angle. (e) The optimal BDA pretilt angle for MDBTN switching.

The ideal tilt angles on the BDA should be between $1^{\circ}$ to $5^{\circ}$ so that the $U \rightarrow T$ or $T \rightarrow U$ transition can function properly, as shown in Fig. 2(e).

Figure 3(a) shows the calculated time dependent transmission of the LC cell with alternating strong and weak electrical pulses. Obviously, bistable switching is achieved. Different from conventional BTN switching mechanism, our approach does not involve any surface anchoring breaking effect. Therefore, the constraints on the cell gap and the requirement of weak anchoring alignment materials can be eliminated.

In order to verify the calculated switching mechanism, a $2.5 \mu \mathrm{m}$ cell gap MD-BTN device was fabricated. LC MLC6204-000 from Merck was used. The upper indium tin oxide glass substrate was coated with a regular rubbed polyimide alignment layer with a pretilt angle of $8^{\circ}$. Photoalignment material was used to generate the BDA surface. By exposing 

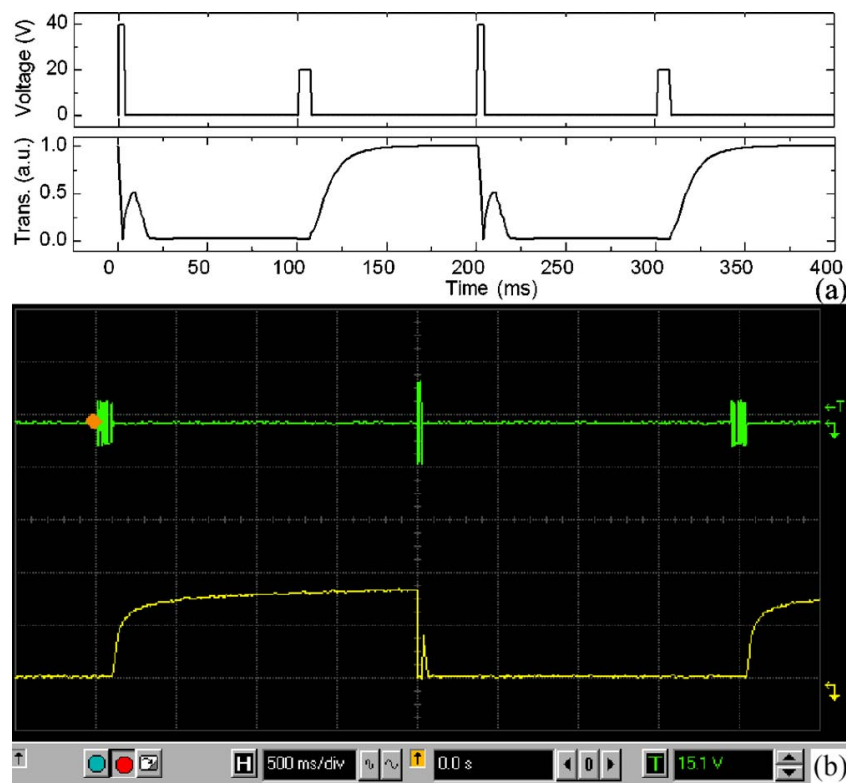

FIG. 3. (Color online) (a) Simulated time dependence of optical transmission of the LC cell under alternating high and low voltage driving. (b) Measured switching dynamics for the experimental MD-BTN cell.

the photoaligned layer twice through a shadow mask at different incident angles, it was easy to obtain two opposite alignment directions of $0.7^{\circ}$ and $179.3^{\circ}$. The domain sizes were $D_{1}=D_{2}=1.5 \mu \mathrm{m}$. The anchoring energy was measured by the standard high voltage technique ${ }^{9}$ to be $1 \mathrm{~mJ} / \mathrm{m}^{2}$. This is quite strong anchoring and is comparable to rubbed polyimide. Obviously, surface anchoring breaking type BTN is not possible with such alignment layers. ${ }^{4}$ Figure 3(b) shows the optical response of this fabricated device. The upper curve shows the driving signal and the lower curve shows the transmittance. The polarizer and analyzer angles are $+45^{\circ}$ and $-45^{\circ}$ to the input LC director, respectively. The measured result is exactly as predicted by the dynamic simulation results in Fig. 3(a). This shows that the theoretical formulation of the competing dynamic flow effect is correct. Indeed the backflow can be controlled using this new BDA surface. Figure 4 shows a passive matrix MD-BTN display with a resolution of $64 \times 64$. It has permanent bistability with no decay of transmittance for a long period of time. The driving method is the same as the conventional passive matrix driving method.

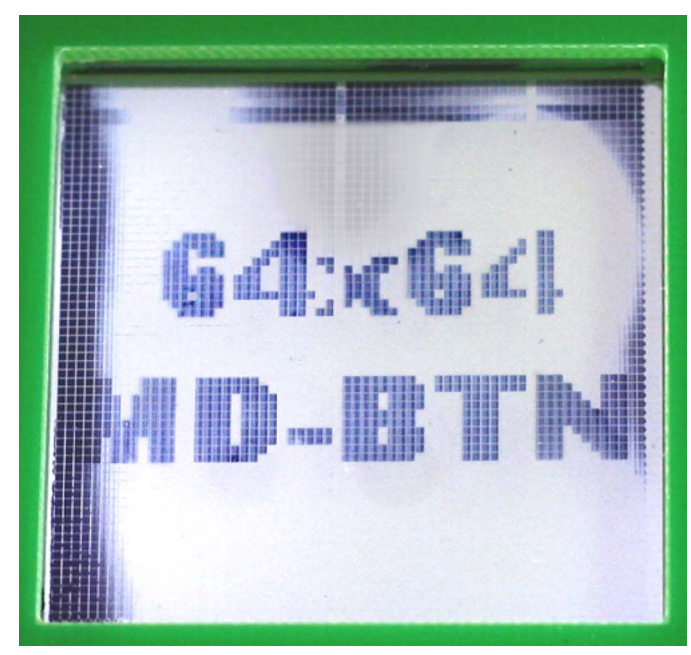

FIG. 4. (Color online) A permanently stable $64 \times 64$ passive matrix MD-BTN display.

In conclusion, we have investigated a new type of alignment surface with alternating bidirectional pretilt. This BDA has an interesting effect on the dynamic flow of the LC system. It is a new approach to control the dynamic response of the LC display. The dynamic flow of the LC molecule consists of two components. The resultant flow direction can be controlled by the applied voltage. We applied this BDA to the case of BTN. This MD-BTN shows true bistability without complicated anchoring breaking requirements. We envisage other applications for this BDA as well.

This research is supported by Hong Kong Research Grants Council Grant No. 614905.

${ }^{1}$ C. Z. Van Doorn, J. Phys. (Paris), Colloq. 36, C1-261 (1975).

${ }^{2}$ D. W. Berreman, J. Appl. Phys. 46, 3746 (1975).

${ }^{3}$ P. Bos and K. Koehler, Mol. Cryst. Liq. Cryst. 113, 329 (1984).

${ }^{4}$ I. Dozov, M. Nobili, and G. Durand, Appl. Phys. Lett. 70, 1179 (1997).

${ }^{5}$ J. T. K. Wan, O. K. C. Tsui, H.-S. Kwok, and P. Sheng, Phys. Rev. E 72, 021711 (2005).

${ }^{6}$ J. L. Ericksen, Trans. Soc. Rheol. 5, 23 (1961).

${ }^{7}$ F. M. Leslie, Q. J. Mech. Appl. Math. 19, 357 (1966).

${ }^{8}$ I. W. Stewart, The Static and Dynamic Continuum Theory of Liquid Crystals (Taylor \& Francis, London, 2004).

${ }^{9}$ Yu. A. Nastishin, R. D. Polak, and S. V. Shiyanovskii, Appl. Phys. Lett. 75, 202 (1999) 